\title{
Análisis de la relación Estado moderno y capital: aproximaciones metodológicas ${ }^{1}$ \\ Analysis of the relationship between the modern state and capital: methodological approaches
}

\author{
Yessenia Fallas Jiménez \\ Escuela de Trabajo Social, \\ Universidad de Costa Rica, San José, Costa Rica \\ yessenia.fallasjimenez@ucr.ac.cr \\ https://orcid.org/0000-0003-4345-5657
}

\section{Resumen}

Introducción: Este texto forma parte de los resultados del proyecto de Investigación Fundamentos históricos de la génesis del Estado moderno y su relación con la producción capitalista. Esta investigación permitió indagar las determinaciones históricas que conforman la relación entre Estado moderno y capital, entendiendo que la configuración de los elementos que permitieron la ascensión del capital requirieron de la existencia de una estructura política que acompañara el proceso de acumulación.

Objetivo: uno de los objetivos específicos de la investigación establece lo siguiente: «Elaborar una propuesta de fundamentación teórica para la comprensión crítica acerca de la producción capitalista, el Estado moderno y la relación histórica entre ambos». Como derivación de este objetivo se estudiaron algunos elementos de carácter metodológico que orientan el análisis del Estado y su relación con la producción capitalista.

Método: dada la naturaleza de la investigación, se recurrió al análisis de fuentes secundarias orientadas a la discusión de los procesos históricos referidos al Estado y a la producción capitalista, así como a bibliografía propia de la producción teórica marxista para identificar los debates existentes.

Resultados: existe una serie de elementos metodológicos básicos que se convierten en propuestas de abordaje y comprensión de ambas categorías y que están vinculados a los procesos históricos que les dan origen a ambas.

Conclusiones: es necesario entender los objetos de estudio a partir de procesos históricos más amplios, identificando la forma en que las mediaciones con la historia general se configuran como constituyentes contradictorios de dichos objetos.

Palabras clave: Capitalismo, Producción, Acumulación, Política, Sociedad.

1 Este texto fue presentado como ponencia en la Jornada de Investigación de la Escuela de Trabajo Social 2021 denominada Tendencias Contemporáneas de la Investigación en Trabajo social. 


\begin{abstract}
Introduction: This text is part of the results of the Research project Historical foundations of the genesis of the modern State and its relationship with capitalist production. This research allowed to investigate the historical determinations that shape the relationship between modern State and capital, understanding that the configuration of the elements that allowed the rise of capital required the existence of a political structure that accompanied the accumulation process.

Objective: One of the specific objectives of the research establishes the following: "To elaborate a proposal of theoretical foundation for the critical understanding about capitalist production, the modern State and the historical relationship between both". As a derivation of this objective, some elements of methodological character that guide the analysis of the State and its relation with capitalist production were studied.

Method: Given the nature of the research, we resorted to the analysis of secondary sources oriented to the discussion of historical processes referred to the State and capitalist production, as well as to bibliography proper to Marxist theoretical production in order to identify existing debates.

Results: There is a series of basic methodological elements that become proposals for the approach and understanding of both categories and that are linked to the historical processes that give rise to both.

Conclusions: It is necessary to understand the objects of study from broader historical processes, identifying the way in which the mediations with the general history are configured as contradictory constituents of said objects.
\end{abstract}

Keywords: Capitalism, Production, Accumulation, Politics, Society.

\title{
Introducción
}

La experiencia formativa de la Escuela de Trabajo Social de la Universidad de Costa Rica tiene como elemento central la discusión sobre la reproducción del Estado moderno. El hecho de que, como profesión, el trabajo social se encuentre orgánicamente articulado a las formas en que el Estado moderno se reproduce y desarrolla sus funciones históricas en la sociedad burguesa hace que la reflexión sobre las particularidades de esta estructura política llamada Estado moderno sea un elemento fundamental en la formación profesional.

Como una derivación de esta discusión, las reflexiones acerca de la configuración de la política pública y en especial de la política social son también centrales en la formación profesional. Esto se expresa en el hecho de que según el Plan de Estudios de la Escuela de Trabajo Social-UCR $(2019$, 1), cuatro de los seis cursos de práctica sean 
directamente una forma de acercamiento a la planificación y ejecución de la política pública: Taller III y IV: Gestión de servicios sociales y Taller V y VI: Análisis y diseño de servicios sociales y los otros dos, aunque no tengan la centralidad en dicho tema, necesariamente se aproximan a la política pública local dada la naturaleza de las discusiones que pretenden abordar, a saber Taller I y II: Organización local y construcción de ciudadanía.

No corresponde detallar en este momento la cantidad de cursos de la malla curricular en la que la discusión acerca del Estado, la política pública y social aparece como tema de la formación, más bien lo que interesa es mostrar que dada la naturaleza de la profesión, este conjunto de categorías tiene una fuerte presencia a lo largo de la formación profesional.

En esta misma línea es posible observar que los objetos, temas y discusiones contenidos en los cursos de la malla curricular incorporan predominantemente la discusión de la política pública y, por lo tanto, del Estado. Esta es sin duda una apuesta pedagógica y teórico-política de la Escuela de Trabajo Social a la hora de desarrollar sus procesos formativos, pues ella se funda en la concepción de que la génesis, la naturaleza y por lo tanto la reproducción de la profesión está orgánicamente articulada a la configuración histórica del Estado moderno y a la función social que el mismo desempeña, entendiendo al mismo tiempo que esto necesariamente demanda una comprensión de las mediaciones económicas y políticas que lo constituyen como producto histórico y a las particularidades que caracterizan su desarrollo contradictorio.

No es por tanto una casualidad que la reflexión sobre el Estado y las políticas públicas y sociales sea una constante en la investigación dentro de la Escuela, así por ejemplo de los 18 trabajos finales de graduación defendidos en el año 2020 ocho giran alrededor del Estado y la política pública (Escuela de Trabajo Social, ETSoc. 2021.)

Es por lo anterior que parece necesario trazar algunos elementos básicos para el abordaje metodológico de la categoría Estado moderno. No es la finalidad de este texto señalar especificidades referidas a las categorías política pública y política social, pero es claro que, al identificar los puntos de partida para la reflexión sobre el Estado, se están trazando también algunos elementos básicos para la discusión de ambas.

\section{Los aspectos metodológicos centrales para el estudio de la relación entre estado moderno y capital}

Cabe aclarar en este punto que, al hablar de elementos metodológicos, no se hace referencia a elementos de carácter técnico u operativo para su estudio, sino más bien a una reflexión acerca del método en cuanto una forma de establecer y desarrollar una relación sujeto-objeto que supera esos asuntos técnicos para reproducirse en el ámbito filosófico y ético-político. 
La forma en la que se estudia y por lo tanto se comprende la categoría Estado moderno refleja una determinada perspectiva teórica que puede reproducir las formas más conservadoras de comprensión, a saber: deshistorizadas y naturalizantes o bien, entenderlas como elementos articulados a procesos históricos más complejos, que en la sociedad burguesa refieren específicamente al proceso de acumulación de capital. Al hablar de la relación sujeto-objeto, los aspectos referidos al método forman parte del movimiento de reproducción racional de la realidad, al mismo tiempo esa reproducción racional no queda limitada a un abordaje conceptual de las categorías históricas, que se reproducen sin consecuencias materiales para las relaciones sociales.

Por el contrario, esa reproducción puede legitimar la forma social predominante o bien, ejercer una función de crítica (radical o no) de dicha forma social, en palabras de Netto $(2012,55)$ la teoría es «la reproducción ideal del movimiento real del objeto por el sujeto que investiga: por la teoría, el sujeto reproduce en su pensamiento la estructura y dinámica del objeto que investiga. Y esta reproducción (que constituye propiamente el conocimiento teórico) será tanto más correcta y verdadera cuanto más fiel el sujeto sea al objeto».

Este último elemento es lo que otorga un peso ético-político a la forma en la que la relación sujeto-objeto se desarrolla, pues las escogencias políticas de los sujetos que conocen-investigan pueden articularse a formulaciones teóricas que legitiman la forma de ser de los objetos estudiados y por tanto de la forma social capitalista que los contiene. Asimismo, pueden establecer elaboraciones que produzcan no solamente una comprensión histórica de esos objetos, sino una crítica al mundo en el que el mismo existe.

A partir de lo anterior, pueden señalarse algunas consideraciones metodológicas básicas para el desarrollo de un análisis de la categoría Estado moderno cuya finalidad sea entender su reproducción como parte de un proceso histórico articulado orgánicamente al proceso de reproducción ampliada de capital, entendiendo con ello que las transformaciones en ese proceso de acumulación se configuran como mediaciones constitutivas de la formación del Estado moderno.

1. Hablar del Estado moderno desde la perspectiva que se propone el presente texto demanda necesariamente hablar del capital, más específicamente del proceso de acumulación de capital. En este sentido, parece ser necesario entender, en primer lugar, al Estado moderno como un producto histórico que surge en el momento en el que el capital alcanza su plena madurez. El Estado moderno como estructura política parece acompañar de forma complementaria el momento histórico en el que el capital productivo se coloca como la forma hegemónica de acumulación de capital y base de toda producción social.

El capital es una relación social y como tal, se caracteriza por ser una relación que tiene como fundamento la producción de mercancías, al mismo tiempo esa producción es el mecanismo más eficiente para la acumulación de valor. El capital en cuanto relación social 
se caracteriza por la existencia de un imperativo que lo define como relación y lo impulsa a constituirse en un modo absoluto de control de todas las relaciones sociales, ese imperativo refiere a la necesidad de acumulación siempre creciente de valor.

Como relación social y modo de control de las relaciones sociales el capital debió constituirse durante su historia en la forma predominante de desenvolvimiento económico a lo largo y ancho del planeta, incorporando dentro de su proceso de reproducción todas las relaciones sociales ya existentes, o creando nuevas formas que contribuyeran a la realización de su imperativo.

Si se compara la producción capitalista de capital con sus formas embrionarias en la forma de capital mercantil y usurero (Marx 2011) se observa que la relación entre las dimensiones internas (producción, circulación y consumo) que constituyen las unidades básicas de producción de esas formas anteriores se caracterizaron por la existencia de una autosuficiencia que se rompe con el modo de producción capitalista. En este, la relación entre esas dimensiones no se desarrolla a partir de los parámetros de la autosuficiencia y además no puede hacerlo, pues el capital no tolera ninguna forma de control, ya que en este modo de producción no es la satisfacción de las necesidades humanas, sino el imperativo de la acumulación lo que define el desarrollo del proceso productivo y lo que de él resulte:

Como potencial productor de valores históricamente específico, el capital no puede ser actualizado y "realizado" (y simultáneamente también reproducido en una forma ampliada gracias a su "realización"), sin entrar en los dominios de la circulación. La relación entre la producción y el consumo es así redefinida radicalmente dentro de su marco de referencia, de tal manera que la muy requerida unidad de ambos se torna insuperablemente problemática, lo que trae consigo con el paso del tiempo también la necesidad de una crisis de un tipo o de otro. Esta vulnerabilidad a las vicisitudes de la circulación es una determinación crucial a la cual ninguna "economía doméstica" de la antigüedad, y muchísimo menos la Edad Media -para no hablar de las unidades reproductivas socioeconómicas del comunismo primitivo y de las antiguas ciudades coloniales a las que se refería Marx en algunas de sus obras principales - debió someterse, dado que ellas estaban orientadas primordialmente hacia la producción y el consumo directos del valor de uso (Mészáros 2001, 52-53).

La autosuficiencia propia de modos de producción anteriores definía los parámetros del proceso productivo, es decir, de la relación con la naturaleza y de las relaciones entre los sujetos resultante de ella. Al mismo tiempo, definía las condiciones de la circulación y el consumo, pues en los modos anteriores el fundamento de la producción no era la acumulación de valor y por tanto el desarrollo de las fuerzas productivas, acompañaba las limitaciones que la regulación autosuficiente entre esas tres dimensiones le colocaba al proceso productivo (en otras palabras se producía lo que se necesitaba y la forma en la que 
eso circulaba dependía de la necesidad sobre la cual descansaba la producción específica de valores de uso).

La superación de los obstáculos de la autosuficiencia que caracterizó los modos de producción anteriores fue muy favorable para el proceso de acumulación, esto porque marcó el impulso reproductivo del sistema y su capacidad de expansión, pues el límite de la producción y la circulación de valores de uso ya no está definido por la satisfacción de las necesidades y, por lo tanto, puede producirse todo lo que sea posible. Sin embargo, al mismo tiempo esa superación trajo consigo la inevitable pérdida de control para el propio capital, lo que quiere decir que dado su imperativo de acumulación y el rompimiento de los obstáculos dados por la autosuficiencia (que con el tiempo se convirtieron en «defectos estructurales»), el capital no tiene cómo colocar controles a pesar de que su realización implique la propia destrucción de la naturaleza y de los seres humanos (Mészáros 2001).

A pesar de que esta forma de producción material es incontrolable, es posible observar que en un determinado momento de su desarrollo, justamente en el periodo en que el modo de producción capitalista inicia su tránsito hacia el control de todas las formas productivas en el planeta, el Estado moderno surge como una estructura política, no solo para ejercer funciones en el plano político, sino para tratar de remediar los defectos que surgen por la falta de autosuficiencia a lo interno de las unidades productivas del sistema. Dado lo anterior:

resulta así de lo más revelador que el estado moderno deba emerger con la misma inexorabilidad que caracteriza la triunfante difusión de las estructuras económicas del capital, complementándolas como la estructura de mando política totalizadora del capital. Tal despliegue inexorable de las estructuras estrechamente intervinculadas del capital en todas las esferas es esencial para establecer la condicionada viabilidad de este singular modo de control metabólico social a lo largo de su existencia histórica.

La formación del estado moderno es un requerimiento absoluto para asegurar y resguardar sobre base permanente los logros productivos del sistema. La llegada del capital al predominio en el ámbito de la producción material y el desarrollo de las prácticas políticas totalizadoras en la forma del estado moderno van de la mano (Mészáros 2001, 57).

Así, el Estado moderno surge en el propio proceso histórico como la estructura política específica del capital ${ }^{2}$. Como forma de control de las relaciones sociales, el capital funda su control económico en la división jerárquica del trabajo y su respectiva división en clases sociales en la que él mismo ejerce el mando, pero en la esfera política el Estado moderno se configura como la estructura del control, necesaria para un sistema de relaciones en las que todo debe someterse a los imperativos de la producción capitalista.

2 Lo que de ninguna manera significa que en modos de producción anteriores no hayan existido otras estructuras políticas (incluso en la forma de Estado) propias de las sociedades divididas en clases sociales. 
Así, a lo largo de la historia y particularmente del siglo XX, observamos que las funciones históricas del Estado moderno acompañan de forma cercana el proceso de acumulación de capital y sus transformaciones específicas, como veremos más adelante.

2. Por esta razón, el análisis del Estado moderno demanda necesariamente entender qué es el capital y qué es la producción capitalista como categorías históricas, sin las cuales la conformación de una estructura política llamada Estado moderno no sería posible. En ese sentido, vale la pena recordar que el capital es una relación social, esto es fundamental no solamente para entender que no siempre existió como forma predominante de control de las relaciones sociales y que por lo tanto tampoco puede pensarse como la única alternativa para el futuro de la humanidad. Así, si observamos las condiciones históricas que acompañaron la conformación del capital como una relación social que alcanza su mayor grado de madurez con la producción capitalista, observamos también la capacidad de someter a su propia lógica todas las relaciones (y mediaciones de segundo orden) ${ }^{3}$ que le anteceden, incluyendo los aspectos culturales, ideológicos, las instituciones, entre otras, y que le fueron funcionales a su lógica reproductiva o bien, aprovecharse de su dominio material para asegurar la configuración de otras que se sumaran a su predominio, ante lo que Mészáros $(2001,124)$ va a indicar que:

En relación con la manera en están vinculados que todos estos constituyentes del modo de control metabólico social, no podemos hablar más que de un círculo vicioso. Porque las mediaciones de segundo orden particulares El autor se refiere aqui entre otras mediaciones de segundo orden a la familiar nuclear, los medios de producción alienados, el dinero, los objetivos de la producción fetichista, el trabajo separado de la posibilidad de control, las diversas formas de Estado del capital y el mercado mundial, se sostienen recíprocamente unas a otras, haciendo imposible contrarrestar la fuerza alienante y paralizadora de cada una de ellas por separado mientras se deja intacto el inmenso poder de autoregeneración y auto-imposición del sistema en su conjunto.

Este aspecto es fundamental, no como premisa fatalista que reproduce el discurso neoliberal y cierra toda posibilidad a un orden social más allá del capital, sino como constatación histórica de que a lo largo de muchos siglos el capital se constituyó en un sistema de control de las relaciones sociales, un producto histórico, por lo tanto su naturaleza es histórica, lo que asegura la posibilidad de que, llegado el momento y configuradas las debidas fuerzas sociales contrarias, el mismo puede ser superado. No corresponde aquí entrar en los debates acerca de las estrategias de superación del capital, pero sí es importante recordar que, dadas las actuales condiciones de la producción capitalista, la respuesta al desafío de superación del capital es una tarea que la humanidad

3 Debe recordarse que la mediación primaria hace referencia a la relación ser social-naturaleza, es decir, a la transformación de la naturaleza para la producción de valores de uso. 
deberá colocarse más temprano que tarde, en el entendido de que lo único que el capital tiene para ofrecer es deshumanización.

3. Esta estructura política en la forma de Estado moderno cumple una determinada función social que se desarrolla en torno al proceso de acumulación. En relación con lo anterior es fundamental preguntarse ¿cuál es su función social? (lo que demanda necesariamente reflexionar sobre la naturaleza del Estado moderno) y ¿cómo esa función social se expresa a lo largo de su desarrollo histórico?

No es el objetivo de este texto analizar las formas en las que dicha función se desarrolla, pero sí es necesario apuntar al menos tres elementos. En primer lugar, en el plano económico, el Estado realiza una función indispensable en el proceso de acumulación: al mismo tiempo que es un potente consumidor de mercancías, el Estado también contribuye en la preparación de fuerza de trabajo a través de la formación de contingentes de trabajadores y trabajadoras que desatollarán los procesos productivos especialmente en aquellas áreas donde la fuerza de trabajo podría escasear. Obsérvese que es común que, en la organización de los procesos formativos, el Estado garantiza la preparación técnica de masas de trabajadores y trabajadoras pertenecientes a aquellas camadas de la clase trabajadora que además de ser formadas en instituciones públicas estarán destacadas en las funciones más técnicas de la producción y de los servicios, y no necesariamente en aquellas que demandan mayores niveles de formación profesionaluniversitaria. El Estado garantiza la existencia de una masa de personas que pueda asumir los procesos menos calificados que al mismo tiempo serán los que tengan salarios más bajos en el mercado laboral.

Asimismo, la preparación de la fuerza de trabajo junto con otras acciones estatales referidas a la seguridad social y la asistencia, a pesar de que después de la década de 1980 han sufrido un proceso de focalización, juega también un papel fundamental en el proceso de producción de plusvalía relativa, empujando hacia abajo el valor de las fuerzas de trabajo. Este proceso tuvo, junto con las políticas de pleno empleo, un papel fundamental en la recuperación de la crisis de 1929, particularmente en los países centrales en la forma del llamado Estado de Bienestar, momento en el que la bonanza económica producto de la acumulación generada por el complejo militar industrial sirvió como base material para el desarrollo de acciones proteccionistas.

Finalmente, es importante recordar que la estructura política del Estado moderno es fundamental en el establecimiento de normativas nacionales e internacionales para la regulación de la circulación y el consumo de mercancías. Un ejemplo de ello es el hecho de que es en el seno de los parlamentos donde se definen los procesos de apertura comercial (a través de tratados de libre comercio), regulación de los monopolios tanto públicos como privados de acuerdo con las necesidades del proceso de acumulación, flexibilización de las 
regulaciones tributarias que impactan también dicho proceso, así como acciones de rescate estatal para empresas que enfrentan crisis económicas.

En segundo lugar, en el plano de lo político, el Estado moderno tiene una función esencial, por un lado, asegura el ordenamiento de las relaciones de poder entre diferentes sectores a lo interno de cada clase y, por otro, legitima mediante la institucionalidad democrática las relaciones de poder entre esos sectores, lo que garantiza una organización del poder que no atente contra los intereses del proceso de acumulación. La separación entre la estructura de control económica y la estructura de control política no juega de ninguna manera una doble contabilidad en el plano más amplio del proceso de acumulación; por el contrario, garantiza que sea posible, mediante mecanismos ampliamente legitimados, que aquellos sectores con acceso a la arena de lucha electoral y la administración del Estado lo hagan sin atentar contra el curso general del proceso de acumulación (excepto cuando ese mismo proceso demanda acciones económicas que el partido político en la administración del Estado se opone a ejecutar: como lo fue el Chile de Allende).

Por último, en el plano ideológico, el Estado reproduce las contradicciones de una estructura que, siempre que sea posible, incorpora lo intereses de la clase trabajadora y operacianaliza políticas y direccionando recursos que buscan atender remedialmente las demandas inmediatas de la clase trabajadora. Todo ello dentro de los límites del propio proceso productivo, por lo tanto, si bien las políticas públicas pueden eventualmente resolver necesidades inmediatas de la clase trabajadora (y debe quedar claro que lo hacen con las camadas más precarizadas de dicha clase, pues la focalización se hizo parte fundamental de las políticas neoliberales a partir de la década de 1980) no pueden bajo ninguna circunstancia resolver los grandes desafíos que la humanidad enfrenta.

Esa imposibilidad no está dada por problemas en la administración o gestión de las políticas públicas, sino por la propia naturaleza del Estado moderno que actúa dentro de los límites del proceso de acumulación. Vale la pena mencionar en este punto que es justamente este elemento el que señala los límites del reformismo, pues la ampliación de políticas sociales durante las décadas de 1940-1960 fue posible en tanto contribuyeron al saludable proceso de acumulación y pocas décadas después mostraron no solamente la imposibilidad de expansión universal de dichas políticas (tanto para el centro como para la periferia capitalista), sino también su naturaleza altamente regresiva, porque una vez llegada la crisis estructural del capital hubo un desmonte de las conquistas y concesiones otorgadas a la clase trabajadora en años anteriores. En tal sentido,

Los excedentes económicos disponibles y las estrategias reformistas de las nuevas élites políticas (políticos profesionales) abrieron la posibilidad de graduales conquistas económicas y políticas de parte de los trabajadores, a través de la lucha sindical y de pactos políticos (...) 
Estas transformaciones ampliaron el Estado tanto en relación a su base representativa -inicialmente con la participación electoral de los trabajadores, y después, de las mujeres- como también en relación con sus funciones económicas y sociales. Además de proteger los derechos de los propietarios, las instituciones estatales pasaron a garantizar complementariamente los derechos sociales de los no-propietarios. Con eso, los Estados Nacionales pasaron a intervenir y a regular cada vez más las relaciones entre capital y trabajo, por medio de nuevas leyes e instituciones de políticas públicas proveedoras de servicios sociales, financiados a través de la creación de nuevos impuestos (...) Como dice Hobsbawm (1995), el capitalismo fue obligado a demostrar al mundo que pleno empleo, bienestar y democracia eran posibles sin revolución social. Éste parecía ser el único modo, en aquel momento, de detener el avance de las ideas socialistas y comunistas entre las clases subalternas (Batista de Abreu 2003, 75).

Lo que a su vez explica por qué una vez llegada la crisis estructural del capital fue necesaria contraer el avance de dichas políticas, junto con el traslado de los negocios más rentables en la producción de valor que estaban en manos del Estado hacia el sector privado.

4. A raíz de los tres elementos históricos anteriores surge otro de carácter teóricopolítico, a saber: la necesaria crítica a las formas predominantes de comprensión del Estado (y la política social) que van desde la reducciones que lo colocan como una estructura imparcial que incorpora indistintamente intereses de los fragmentos de clase que están en su administración hasta aquellas que lo entienden como una estructura política cuya función social está orientada a la consecución del bien común.

Este elemento es fundamental, pues si bien el Estado moderno es una estructura contradictoria que en diferentes momentos y motivado por fuerzas sociales diversas (el proceso de acumulación, el movimiento social o la concesión de la clase hegemónica) incorpora demandas de sectores de la clase trabajadora, los límites de dicha incorporación vienen dados por el significado histórico que esas demandas tienen en relación con la acumulación de valor. Así, nada que desafíe la naturaleza desigual de la relación capital puede incorporarse a la lógica de actuación del Estado moderno, porque las alternativas superadoras del capital no pueden entrar en los límites dentro de los cuales él mismo se reproduce y realiza su imperativo estructural.

En ese sentido, vale la pena reflexionar sobre el hecho de que «sin embargo, bajo la presión de la crisis estructural, el capital ya no pudo ofrecer ninguna ganancia significativa al interlocutor racional, sino que, por el contrario, se vio obligado a retomar concesiones pasadas, atacando sin piedad los mismos cimientos del estado de bienestar. como las garantías legales para la protección y defensa de la clase trabajadora a través de un conjunto de leyes autoritarias contra el movimiento sindical, todas aprobadas democráticamente, y el 
orden político establecido tuvo que renunciar a su legitimidad, exponiendo, al mismo tiempo, la imposibilidad de la posición defensiva del movimiento obrero» ${ }^{4}$ (Mészáros 2001, 24).

Si se entiende que la naturaleza del Estado moderno está orgánicamente articulada a la naturaleza del capital tal y como se mencionó en el inicio, se entenderá también que las determinaciones que definen la economía social, que en este caso tienen la división jerárquica del trabajo como aspecto fundamental, son también las que definen la estructura política que le acompaña, en palabras de Marx $(2012,76)$,

El Estado jamás descubrirá en "el Estado y la organización de la sociedad”, (...) la razón de los males sociales. En todas partes donde hay partidos políticos, cada uno de ellos halla la razón de cada mal en el hecho de que su adversario ocupa su lugar en la dirección del Estado. Incluso los políticos radicales y revolucionarios encuentran la razón no en la esencia [Wesen] del Estado, sino en una forma determinada de Estado que pretenden reemplazar por otra.

Desde el punto de vista político, el Estado y la organización de la sociedad no son dos cosas diferentes. El Estado es la organización de la sociedad. En la medida en que el Estado reconoce la existencia de anomalías sociales, busca la razón de las mismas ya sea en las leyes naturales que ninguna fuerza humana puede doblegar, o en la vida privada que es independiente del Estado, o bien en una inadaptación de la administración que depende del Estado.

Finalmente, debe destacarse un elemento central para el trabajo social, el mismo refiere a la relación entre el capital, el Estado moderno, la política social y la profesión. No es casualidad que la relación entre estos cuatro elementos sea una preocupación para los contingentes profesionales en formación, porque la misma está plagada de contradicciones que refieren a su origen como profesión y a la naturaleza y función social que tiene en esta sociedad.

Cabe recordar que el origen del trabajo social está orgánicamente articulado a la función social que el Estado moderno ocupa en relación con el proceso de acumulación. Así, tal y como se mencionó al inicio, si el Estado moderno surge en un momento determinado (el de mayor madurez) del desarrollo del capital como relación social, corresponde pensar que el trabajo social surge en el proceso histórico como una categoría profesional cuya necesidad está dada por la materialización de las acciones estatales

4Entretanto, sob a pressão da crise estrutural, o capital não teve mais condições de oferecer qualquer ganho significativo ao interlocutor racional, mas ao contrário, foi obrigado a retomar as concessões passadas, atacando sem piedade as próprias bases do Estado de bem-estar, bem como as salvaguardas legais de proteção e defesa do operariado por meio de um conjunto de leis autoritárias contrárias ao movimento sindical, todas aprovadas democraticamente, e a ordem política estabelecida teve de abrir mão de sua legitimidade, expondo, ao mesmo tempo, a inviabilidade da postura defensiva do movimento operário. 
específicas referidas a la ejecución de políticas públicas en el marco de esa estructura política específica.

Así, si bien es cierto que los rasgos históricos constitutivos del trabajo social no pueden ser difuminados ni apagados por la existencia de voluntades profesionales que se contrapongan a las determinaciones históricas que definen el campo de origen y reproducción de la profesión, es ahí donde la comprensión de los límites de la profesión (que se expresan en la particularidad de los procesos de trabajo) parece ser un elemento que debe ser tratado con sumo cuidado en la formación profesional para no reproducir visiones fatalistas ni mesiánicas de la profesión pues,

el fatalismo y el mesianismo, la práctica social aparece transfigurada por concepciones naturalistas e idealista de la vida social, presentes en la trayectoria del debate intelectual del pensamiento social en la modernidad.

La superación del fatalismo y el mesianismo en el análisis de la práctica social con sus derivaciones en el ejercicio profesional- implica desvendar la propia sociedad que gesta esas concepciones y su crítica teórica radical históricamente construida (Villela Iamamoto 1992,183-184).

\section{A modo de conclusiones}

Entender el plano de la contradicción en la que la profesión se reproduce y, como consecuencia, en la que las personas profesionales desarrollan su cotidiano laboral, es fundamental para administrar las posiciones políticas y finalidades éticas del colectivo profesional, al demarcar el campo de posibilidades y alternativas que su trabajo cotidiano, siempre vinculado a la política pública, pueda tener. Aquí la relación entre las realizaciones inmediatas referidas a la satisfacción de necesidades básicas a través de la asistencia y la seguridad social, las cuales deben ser aprehendidas bajo un ángulo analítico que esté acompañado por la comprensión de las determinaciones más universales de la sociedad burguesa que permitan a la persona profesional entender que, si bien la satisfacción de la necesidad inmediatas es fundamental para la vida de un sujeto, en el plano de la superación de una relación social deshumanizadora como el capital su significado no puede ser suficiente.

Dar un contenido diferente a la relación entre necesidades inmediatas y perspectivas superadoras no es un asunto que se define en el lugar de trabajo de la o el profesional, esta perspectiva superadora no es solamente el resultado de la formación, sino que es una determinación histórica que supera los límites de las cuatro paredes universitarias y se coloca en un plano más amplio, relativo incluso al propio movimiento y organización de la clase trabajadora y de los límites estructurales del capital. 


\section{Referencias}

Batista, de Abreu, Haroldo. 2003. Las nuevas configuraciones del Estado y la sociedad civil. En Servicio Social crítico. Hacia la construcción del nuevo proyecto éticopolítico. São Paulo, Brasil: Cortez Editora.

ETSoc-UCR (Escuela de Trabajo social, Universidad de Costa Rica). 2019. Plan de estudios de la carrera de Trabajo Social. San José, Costa Rica: ETSoc-UCR

2021. Trabajos Finales de Graduación, acceso el 30 de setiembre de 2021, http://www.ts.ucr.ac.cr/bv/tfg-lic.php.

Marx, Karl. 2011. Grundrisse. São Paulo, Brasil: Boitempo editorial. 2012. Glosas Criticas Marginales al artículo: "El rey de Prusia y la reforma social. Por un prusiano". En Páginas malditas Sobre La cuestión judía y otros textos, 6186. Buenos Arires, Argentina: Libros de Anarres.

Mészáros, Itsván. 2001. Más allá del capital. Hacia una teoría de la transición. Caracas, Venezuela: Hernmano Vadell Editores.

2000. Para além do capital. Rumo a uma teoria da transição. São Paulo, Brasil: Boitempo Editorial.

Netto, José Paulo. 2012. Trabajo Social: Crítica de la vida cotidiana y Método en Marx. La Plata, Argentina: Productora del Boulevard.

Villela Iamamoto, Marilda. 1992. Servicio social y división del trabajo. São Paulo, Brasil: Cortez Editora. 